\title{
Knowledge Base Stratification and Merging Based on Degree of Support
}

\author{
Anthony Hunter ${ }^{1}$ and Weiru Liu ${ }^{2}$ \\ 1 Department of Computer Science, University College London, \\ Gower Street, London WC1E 6BT, UK \\ 2 School of Electronics, Electrical Engineering and Computer Science, \\ Queen's University Belfast, Belfast BT7 1NN, UK \\ w. liu@qub.ac.uk
}

\begin{abstract}
Most operators for merging multiple knowledge bases (where each is a set of formulae) aim to produce a knowledge base as output that best reflects the information available in the input. Whilst these operators have some valuable properties, they do not provide explicit information on the degree to which each formula in the output has been, in some sense, supported by the different knowledge bases in the input. To address this, in this paper, we first define the degree of support that a formula receives from input knowledge bases. We then provide two ways of determining formulae which have the highest degree of support in the current collection of formulae in $\mathrm{KBs}$, each of which gives a preference (or priority) over formulae that can be used to stratify the formulae in the output. We formulate these two preference criteria, and present an algorithm that given a set of knowledge bases as input, generates a stratified knowledge base as output. Following this, we define some merging operators based on the stratified base. Logical properties of these operators are investigated and a criterion for selecting merging operators is introduced.
\end{abstract}

\section{Introduction}

The notion of priority (preference) is important in inconsistency-tolerant reasoning (such that for potentially inconsistent knowledge-based systems [B+04], belief updating [Gad88], analyzing inconsistent regulations [BB04]; and analyzing social networks [KG06]). Priorities can be encoded in two different ways, one way is to prioritize these sources according to their reliability and another is to attach priorities to items of knowledge within each source [BDP96]. Both approaches usually would require that information on priorities is available explicitly, especially for the reliability of sources.

Merging operators for multiple knowledge bases therefore can be characterized for whether they are designed to merge flat (e.g., [BKMS92,KLM04]) or stratified knowledge/belief bases (e.g.,[B+98,DDL06]). Often, the result of merging, no matter whether for stratified or for flat knowledge bases, is simply a flat base ${ }^{1}$. However, even if the original knowledge bases are not prioritized, some items of knowledge (i.e., some formulae) receive more support (i.e. more preferred) then others. This information is often ignored when a merged result is obtained.

\footnotetext{
${ }^{1}$ We only consider merging in the context of propositional logic. As for possibilistic logic, the merged result is a new possibilistic knowledge base which can be regarded as prioritized.
} 
The importance of differentiating more preferred (w.r.t. support) from less preferred formulae can be seen in many applications such as in requirements engineering or in analyzing findings from clinical trials. In requirements engineering, common requirements from different sources are in general selected first before resolving conflicting requirements [Dav05]. Assume that there are four stakeholders involved in building a new computer system. Stakeholder A (sales person) prefers the new system to be open and fashionable, stakeholder B (system security) prefers the system to be authorized and not open, stakeholder C (programmer) prefers open and fashionable, and stakeholder D (investor) prefers open, and fashionable and easy to use. We can construct four knowledge bases as follows where $p=$ open, $q=$ fashionable, $s=$ authorized and $r=$ easy to use, $K_{A}=\{p, q\}, K_{B}=\{\neg p, s\}, K_{C}=\{p, q\}$, and $K_{D}=\{p, q \wedge r\}$. A combination method in [BKMS92] generates two possible solutions $M_{1}=\{p, q, q \wedge r, s\}$ and $M_{2}=\{\neg p, q, q \wedge r, s\}$. With these two maximal consistent subsets, it is not possible to decide whether it should be $p$ or $\neg p$ in the merged result, although $p$ has support from three bases. The merging operators proposed in [Kon00] overcome this problem. For instance, one of the three operators in [Kon00] selects $M_{1}=\{p, q, q \wedge r, s\}$ in the above example since this subset is consistent with three of the four original bases. However, current merging operators cannot tell which formulae are more preferred in the merged base. In this example, proposition $p$ (the new system should be open) is directly required by three stakeholders, while proposition $s$ is only supported by one stakeholder, nevertheless, they are all treated equally in the merged knowledge base. Furthermore, $q$ is directly given in two bases and is entailed from another base. This extra information is not retained either after merging. We believe that the degree of support for $p$ (no matter whether directly given or implicitly entailed) from these sources should be reflected in a merged base, so the output of merging also states that $p$ is preferred to $q$ and $q$ is preferred to $s$.

In this paper, we investigate how the above two types of support to a formula can be identified when multiple knowledge bases are present. For this, we first define the degree of support that a formula receives from a profile, we then define both the notion of most primed formulae and the most entailed formulae and propose methods to select them. A formula with the highest degree of support in a set of knowledge bases (a profile) is either a most primed or a most entailed formula in the profile. We also propose an algorithm to stratify the union of formulae from multiple knowledge bases based on the degree of support a formula receives, with the result being a stratified base. A stratified base induces a total preorder relation which ranks a more preferred formula ahead of less preferred ones. The significance of such a base can be shown in several aspects. First, common beliefs (knowledge) from the majority of sources are given higher priorities than other beliefs in a profile. Second, it can be used to determine which merging result is better in terms of retaining more important formulae. Third, it can be taken as a prioritized observation base and merging operators tailored towards such a base can be applied [DDL06].

The paper is organized as follows. The preliminaries are introduced in Section 2. Two conceptualizations of most preferred formulae which have the highest degree of support in the current profile are presented, namely, the most primed and the most entailed formulae in Section 3. A stratification algorithm and its properties are studied 
in Section 4. In Section 5, some new merging operators are proposed and their logical properties are discussed. Comparisons with related work and conclusions are given in Section 6.

\section{Preliminaries}

\subsection{Propositional logic}

We consider standard finite classical propositional logic here. We denote atoms as $p, q, r$, etc. and formulas as $\phi, \psi, \gamma$, etc.. A literal is an atom $p$ or its negation $\neg p$ and is denoted as $l_{i}$. The classical consequence relation is denoted as $\vdash$. An interpretation (possible world), $\omega$, is a function from $\mathcal{A}$ (the set of atoms) to $\{0,1\}$ and is denoted as the conjunction of literals $l_{1} \wedge \ldots \wedge l_{|\mathcal{A}|}$, where $|\mathcal{A}|$ means the cardinality of set $\mathcal{A}$. $\omega$ is a model of a formula $\phi$ iff $\omega(\phi)=1$. Two formulae $\phi$ and $\varphi$ are said to be equivalent (or equal), denoted as $\phi \equiv \varphi$, iff they have the same set of models. In this paper, we say $\phi$ is in $K_{i}$ iff there exists a $\varphi \in K_{i}$ such that $\phi \equiv \varphi$ regardless of their syntax. Also, $\wedge K_{i} \vdash \phi$ denotes $\wedge \varphi_{j} \vdash \phi$ where $\varphi_{j} \in K_{i}$.

A knowledge base, $K$, is a collection of propositional formulae and a knowledge profile, $\mathcal{E}=\left\{K_{1}, \ldots, K_{n}\right\}$ contains a set of knowledge bases which are not necessarily distinct. In the following, we let $\mathcal{E}_{\cup}=K_{1} \cup \ldots \cup K_{n}$ and let $\mathcal{E}_{\cap}=K_{1} \cap \ldots \cap K_{n}$ where $\cup$ and $\cap$ are usual set-based union and intersection operators. A subset $E$ of knowledge profile $\mathcal{E}$ itself in turn is a knowledge profile and $E_{\cup}$ and $E_{\cap}$ are similarly defined. In subsequent sections, we always use $\mathcal{E}$ to denote an original knowledge profile, and use $E$ (possibly with subscript) to denote a subset of $\mathcal{E}$.

\subsection{Stratified knowledge bases}

A stratified knowledge base, also called a ranked knowledge base [Bre04] or a prioritized knowledge base [B+93] models a set of formulae with explicit preferences (or priorities) among the formulae. Let $K$ be a knowledge base containing a set of propositional formulae, $(K, \preceq)$ is a stratified base if there is a total preorder relation $\preceq$ on $K . \preceq$ is a total preorder on $K$ iff for any $\phi, \varphi \in K$, either $\phi \preceq \varphi$ or $\varphi \preceq \phi$ holds. A preorder relation, $\preceq$, is transitive and reflexive and its associated strict preorder relation, $\prec$, is defined as $\phi \prec \varphi$ iff $\phi \preceq \varphi$ but $\varphi \npreceq \phi$. $\phi \preceq \varphi$ is interpreted as $\phi$ is at least as preferred (or plausible) as $\varphi$ and $\phi \prec \varphi$ as $\phi$ is more preferred than $\varphi$. Two preorder relations $\preceq_{1}$ and $\preceq_{2}$ are equivalent, denoted as $\preceq_{1} \preceq_{2}$, if for any two formulae $\phi$ and $\varphi, \phi \preceq_{1} \varphi$ implies $\phi \preceq_{2} \varphi$ and vice versa.

For simplicity, in the following we use $\mathcal{S}_{K}$ to denote a stratified version of a knowledge base $K$ without mentioning the total preorder relation on $K$ and $\mathcal{S}_{K}$ can be equivalently represented as a tuple $\mathcal{S}_{K}=\left\langle S_{1}, \ldots, S_{m}\right\rangle$ such that $S_{i} \neq \emptyset,(i=1, \ldots, m)$ and $S_{i}$ contains all the most preferred elements in $K \backslash\left(\cup_{j=1}^{i-1} S_{j}\right)$ w.r.t $\preceq$, that is, $S_{i}=\{\phi \in$ $K \backslash\left(\cup_{j=1}^{i-1} S_{j}\right)$, s.t., $\left.\forall \varphi \in K \backslash\left(\cup_{j=1}^{i-1} S_{j}\right), \phi \preceq \varphi\right\}$. Each $S_{i}$ is called a stratum of $K$ and index $i$ is the priority level of formulae in $S_{i}$. Therefore, the lower the index is, the more preferred a formula is. A stratified knowledge base $K$ can be inconsistent and the degree of inconsistency of $K$ is defined as $\operatorname{lnc}\left(S_{K}\right)=i, i>0$ s.t. $, \cup_{j=1}^{i-1} S_{j} \nvdash \perp, \cup_{j=1}^{i} S_{j} \vdash \perp$. Let $\mathcal{S}_{K_{1}}=\left\langle S_{1}, \ldots, S_{m}\right\rangle$ and $\mathcal{S}_{K_{2}}=\left\langle T_{1}, \ldots, T_{n}\right\rangle$ be two tuples, the concatenation of them is defined as $\mathcal{S}_{K_{1}} \oplus \mathcal{S}_{K_{2}}=\left\langle S_{1}, \ldots, S_{m}, T_{1}, \ldots, T_{n}\right\rangle$. In particular for any tuple 
$\mathcal{S}_{K}, \mathcal{S}_{K} \oplus\langle\rangle=\langle\rangle \oplus \mathcal{S}_{K}=\mathcal{S}_{K}$. Also, we write $\mathcal{S}_{K_{1}} \approx \mathcal{S}_{K_{2}}$ iff $m=n$ and for each $i$ where $S_{i}=\left\{\beta_{1}, \ldots, \beta_{p}\right\}$ and $T_{i}=\left\{\beta_{1}^{\prime}, \ldots, \beta_{p}^{\prime}\right\}$ it is the case that $\beta_{j}=\beta_{j}^{\prime}$, for $j=1, \ldots, p$.

\section{Most preferred formulae}

To characterize that some formulae are more preferred than others in a collection of knowledge bases, we first define the degree of support of a formula. The rational of this definition is that there could be many ways to define a function from formulae to $[0,1]$ and some of them are not acceptable as formalizing the amount of support a formula gets from a knowledge base. So we only give some constraints about what a degree of support function shall obey, rather than specify a single function.

Definition 1. Let $\mathcal{E}$ be a knowledge profile and let $\phi$ be a formula. A real function $S_{\mathcal{E}}$ is a degree of support function for $\mathcal{E}$ iff it satisfies the following two conditions.

1. If $|\mathcal{E}|=1$ and $\phi \in \mathcal{E}_{\cup}$ then $S_{\mathcal{E}}(\phi)=1$

2. If $\mathcal{E}_{\cup} \forall \perp$ and $\phi \in \mathcal{E}_{\cup}$ then $S_{\mathcal{E}}(\phi)>0$, where $\mathcal{E}_{\cup} \forall \perp$ means $\mathcal{E}_{\cup}$ is consistent.

$\phi$ is a most preferred formula if $\forall \varphi \in \mathcal{E}_{\cup}, a b s\left(S_{\mathcal{E}}(\phi)\right) \geq a b s\left(S_{\mathcal{E}}(\varphi)\right)$ holds, where $a b s\left(S_{\mathcal{E}}(\phi)\right)$ returns the positive value (the absolute value) obtained from $S_{\mathcal{E}}(\phi)$.

This definition contains two constraints. The first says that when a knowledge profile contains a single knowledge base then every formula in the base should have the maximum support, and the second states that when a knowledge profile is consistent then every formula appearing in the profile should have a positive degree of support. We now provide some subsidiary definitions that we will use to define two possible definitions of a degree of support functions. Let $\operatorname{Atoms}\left(\mathcal{E}_{\cup}\right)$ be the set of atoms that appear in the formulae in $\mathcal{E}_{\cup}$. We can use the power set of $\operatorname{Atoms}\left(\mathcal{E}_{\cup}\right)$ to denote the set of interpretations of $\mathcal{E}_{\cup}$. For any $I \subseteq \operatorname{Atoms}\left(\mathcal{E}_{\cup}\right)$, if $\alpha \in I$ then $\alpha$ is true in $I$, otherwise $\alpha$ is false in $I$.

Definition 2. For a set of formulae $X$, the set of models of $X$ in the context of $\mathcal{E}$, denoted $\mathrm{M}_{\mathcal{E}}(X)$, is defined as $\mathrm{M}_{\mathcal{E}}(X)=\left\{I \subseteq \operatorname{Atoms}\left(\mathcal{E}_{\cup}\right) \mid I \models \wedge X\right\}$

Definition 3. Let $K$ be a consistent set of formulae and let $\phi$ be a consistent formula. The degree of entailment of $K$ for $\phi$ in the context of $\mathcal{E}$, denoted $E_{\mathcal{E}}(K, \phi)$, is defined as $E_{\mathcal{E}}(K, \phi)=\frac{\left|\mathrm{M}_{\mathcal{E}}(K \cup\{\phi\})\right|}{\left|\mathrm{M}_{\mathcal{E}}(K)\right|}$.

For instance, let $K=\{p, q \wedge r\}$, then $E_{\mathcal{E}}(K, p)=1, E_{\mathcal{E}}(K, p \wedge q)=1, E_{\mathcal{E}}(K, q \wedge r \wedge$ $s)=\frac{1}{2}$.

The Dalal distance (Hamming distance) between $w_{i}$ and $w_{j}$, denoted Dalal $\left(w_{i}, w_{j}\right)$, is the difference in the number of atoms assigned true (i.e. Dalal $\left(w_{i}, w_{j}\right)=\left|w_{i}-w_{j}\right|+$ $\left.\left|w_{j}-w_{i}\right|\right)$. To evaluate the conflict between two formulae, we take a pair of models, one for each formula, such that the Dalal distance is minimized. The degree of conflict is this distance divided by the maximum possible Dalal distance between a pair of models.

Definition 4. Let $X$ and $Y$ be sets of formulae, each of which is consistent. The set of distances between $X$ and $Y$, denoted $\operatorname{Dist}_{\mathcal{E}}(X, Y)$ is defined as

$$
\operatorname{Dist}_{\mathcal{E}}(X, Y)=\left\{\operatorname{Dalal}\left(w_{x}, w_{y}\right) \mid w_{x} \in M_{\mathcal{E}}(X) \wedge w_{y} \in M_{\mathcal{E}}(Y)\right\}
$$


Definition 5. Let $K$ be a consistent set of formulae and let $\phi$ be a consistent formula. The degree of conflict of $K$ for $\phi$ in the context of $\mathcal{E}$, denoted $C_{\mathcal{E}}(K, \phi)$, is defined as follows:

$$
C_{\mathcal{E}}(K, \phi)=\frac{\operatorname{Min}\left(\operatorname{Dist}_{\mathcal{E}}(K,\{\phi\})\right)}{\left|\operatorname{Atoms}\left(\mathcal{E}_{\cup}\right)\right|}
$$

Once again, let $K=\{p, q \wedge r\}$, then $C_{\mathcal{E}}(K, p)=C_{\mathcal{E}}(K, p \wedge q)=0, C_{\mathcal{E}}(K, q \wedge \neg r \wedge s)=$ $1 / 4$.

We now define two instances of a degree of support.

Definition 6. Let $\mathcal{E}$ be a knowledge profile and let $\phi$ be a formula. The drastic degree of support, denoted $S_{\mathcal{E}}^{d}$, is defined as

$$
S_{\mathcal{E}}^{d}(\phi)=\sum_{K \in \mathcal{E}, E_{\mathcal{E}}(K, \phi)=1} E_{\mathcal{E}}(K, \phi)
$$

Definition 7. Let $\mathcal{E}$ be a knowledge profile and let $\phi$ be a formula. The balanced degree of support, denoted $S_{\mathcal{E}}^{b}$, is defined as

$$
S_{\mathcal{E}}^{b}(\phi)=\sum_{K \in \mathcal{E}} E_{\mathcal{E}}(K, \phi)-\sum_{K \in \mathcal{E}} C_{\mathcal{E}}(K, \phi) .
$$

Proposition 1. Let $\mathcal{E}$ be a knowledge profile and let $\phi$ and $\psi$ be formulae.

1. If $\phi \vdash \psi$ then $S_{\mathcal{E}}^{d}(\phi) \leq S_{\mathcal{E}}^{d}(\psi)$

2. If $\mathcal{E} \nvdash \perp$ and $\phi \vdash \psi$, then $S_{\mathcal{E}}^{b}(\phi) \leq S_{\mathcal{E}}^{b}(\psi)$

Example 1. Let $\mathcal{E}=\left\{K_{A}, K_{B}, K_{C}, K_{D}\right\}$ where $K_{A}=\{p, q\}, K_{B}=\{\neg p, s\}$, $K_{C}=\{p, q\}$, and $K_{D}=\{p, q \wedge r\}$ (as defined in the Introduction). Then the drastic degrees of support and the balanced degree of support for formulae $p, q, \neg p, s$, and $q \wedge r$ respectively are

\begin{tabular}{c|ccccc}
\hline & $p$ & $q$ & $\neg p$ & $s$ & $q \wedge r$ \\
\hline$S_{\mathcal{E}}^{d}(\bullet)$ & 3 & 3 & 1 & 1 & 1 \\
$S_{\mathcal{E}}^{b}(\bullet)$ & $\frac{11}{4}$ & $\frac{7}{2}$ & $\frac{1}{4}$ & $\frac{5}{2}$ & $\frac{9}{4}$ \\
\hline
\end{tabular}

Formulae $p$ and $q$ have the highest drastic degree of support from these bases while $q$ is the only formula in $\mathcal{E}_{\cup}$ that has the highest balanced degree of support. The balanced degrees of support for $p$ and $\neg p$ are both less than their drastic degrees of support because one of them contributed to the degree of conflict of the other. $S_{\mathcal{E}}^{d}(q \wedge r)=1$ is increased to $S_{\mathcal{E}}^{b}(q \wedge r)=9 / 4$ because $q \wedge r$ is partially entailed by $K_{A}$ and $K_{C}$, and it is not in conflict with $K_{B}$. If we consider the drastic degree of support, $p$ and $q$ are among the most preferred formulae. It should be pointed out that $p$ is directly given in three bases while $q$ is given in two and is entailed by another, although they have the same degree of support. We want to differentiate these two types of formulae when their degrees of support are the same, since we believe that $p$ (as an individual statement) holds more support than $q$ (if we assume stakeholder D really prefers that both $q$ and $r$ be true, not just $q$ ).

In this paper, we will only consider the drastic degree of support and we conceptualize these two types of most preferred formulae which have the highest drastic degree of support in the current profile, namely the most primed and the most entailed. 
Definition 8. Let $\mathcal{E}=\left\{K_{1}, \ldots, K_{n}\right\}$ be a knowledge profile. We define a total preorder relation $\preceq_{p}$ on $\mathcal{E}_{\cup}$ as follows.

$$
\begin{aligned}
\forall \phi, \varphi & \in \mathcal{E}_{\cup}, \phi \preceq_{p} \varphi \text { iff } S_{\mathcal{E}}^{d p}(\phi) \geq S_{\mathcal{E}}^{d p}(\varphi) \\
\text { where } S_{\mathcal{E}}^{d p}(\phi)=|\sharp(\mathcal{E})| \text { s.t., } \sharp(\mathcal{E}) & =\left\{K \in \mathcal{E} \mid E_{\mathcal{E}}(K, \phi)=1 \text { and } \phi \in K\right\} .
\end{aligned}
$$

$S_{\mathcal{E}}^{d p}(\phi)$ is a variant of $S_{\mathcal{E}}^{d}(\phi)$ in which we not only require that $E_{\mathcal{E}}(K, \phi)=1$ but also $\phi \in K$, so $S_{\mathcal{E}}^{d p}(\phi)$ is more restricted than $S_{\mathcal{E}}^{d}(\phi)$. For instance, with Example 1, we have $S_{\mathcal{E}}^{d}(q)=3$ whilst $S_{\mathcal{E}}^{d p}(q)=2$, because $q \notin K_{D}$ although $E_{\mathcal{E}}\left(K_{D}, q\right)=1$.

Definition 9. Let $\mathcal{E}=\left\{K_{1}, \ldots, K_{n}\right\}$ be a knowledge profile. $\phi \in \mathcal{E}_{\cup}$ is called a most primed formula in $\mathcal{E}_{\cup}$ iff $\phi \preceq_{p} \varphi, \forall \varphi \in \mathcal{E}_{\cup}$.

The most primed formulae are knowledge profile dependent, since a formula can be a most primed formula in one profile but not in another. For example, if we have a knowledge profile $\mathcal{E}=\left\{K_{1}, K_{2}, K_{3}\right\}$ such that $K_{1}=\{p, q, r\}, K_{2}=\{p, r, s\}$, and $K_{3}=\{p, q, \neg r\}$, then the most primed formula in $\mathcal{E}_{\cup}$ is $p$. However, if we delete $p$ from $K_{3}$, then the most primed formulae are $\{p, q, r\}$.

Definition 10. Let $\mathcal{E}=\left\{K_{1}, \ldots, K_{n}\right\}$ be a knowledge profile. We define a total preorder relation $\preceq_{e}$ on $\mathcal{E}_{\cup}$ as follows

$$
\forall \phi, \varphi \in \mathcal{E}_{\cup}, \phi \preceq_{e} \varphi \text { iff } S_{\mathcal{E}}^{d}(\phi) \geq S_{\mathcal{E}}^{d}(\varphi)
$$

$\phi$ is a most entailed formula in $\mathcal{E}_{\cup}$ iff $\forall \psi \in \mathcal{E}_{\cup}, \phi \preceq_{e} \varphi$.

Like the most primed formulae in $\mathcal{E}_{\cup}$, the most entailed formulae are dependent on which knowledge profile is under consideration. A formula can be a most entailed formula in one knowledge profile but is not in another.

It is obvious that given $\mathcal{E}=\left\{K_{1}, \ldots, K_{n}\right\}, \forall \phi \in \mathcal{E}_{\cup}, S_{\mathcal{E}}^{d p}(\phi) \leq S_{\mathcal{E}}^{d}(\phi)$.

Example 2. Let $\mathcal{E}_{1}=\left\{K_{1}, K_{2}, K_{3}\right\}$ with $K_{1}=\{p, q\}, K_{2}=\{p \wedge q, r\}$ and $K_{3}=$ $\{p, s\}$, then, $S_{\mathcal{E}}^{d}(p)=3$ and $S_{\mathcal{E}}^{d p}(p)=2, S_{\mathcal{E}}^{d}(q)=2$ and $S_{\mathcal{E}}^{d p}(q)=1$, and $S_{\mathcal{E}}^{d}(r)=$ $S_{\mathcal{E}}^{d p}(r)=1, S_{\mathcal{E}}^{d}(s)=S_{\mathcal{E}}^{d p}(s)=1$.

It should be noted that although the concept of most entailed formulae subsumes the concept of most primed formulae, we still prefer to have these two types of preferences defined separately. One advantage of this is that we would be able to distinguish a formula that is directly given by several knowledge bases (that is, this element of knowledge is explicitly believed and supported) from a formula which is inferred from the same number of knowledge bases (that is, this element of knowledge is implicitly believed and supported), if these two formulae have the same degree of support. For instance, for $p$ and $q$ in Example 1 we have $S_{\mathcal{E}}^{d}(p)=S_{\mathcal{E}}^{d}(q)=3$, however, $p$ holds more confidence in the four knowledge bases than $q$ does. Therefore, we believe that $p$ is more preferred than $q$ in the profile and this rationale is used in the algorithm in the following section. 


\section{Stratification of a Knowledge Profile}

The algorithm below ranks a more preferred formula (using the most primed and the most entailed formulae) ahead of a less preferred formula. $\mathrm{S}_{\mathrm{MPE}}(\mathcal{E})$ stands for an algorithm for Stratification based on the Most Primed and/or Entailed formulae in $\mathcal{E}_{\cup}$.

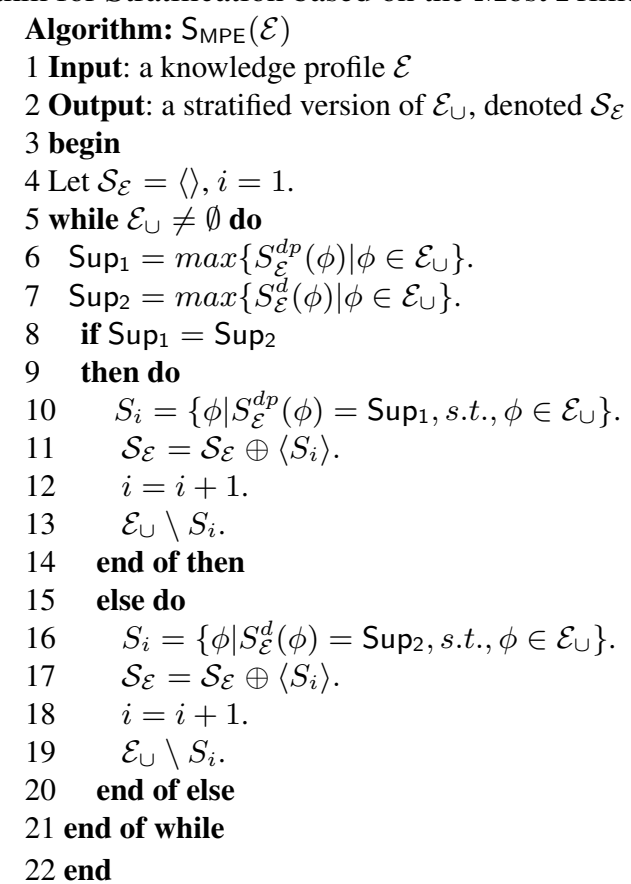

Example 3. Let $\mathcal{E}=\left\{K_{1}, \ldots, K_{7}\right\}$ where $K_{1}=K_{2}=K_{3}=\{p\}, K_{4}=K_{5}=K_{6}=$ $\{q \wedge r\}$ and $K_{7}=\{p, s\}$. Then $\mathrm{S}_{\mathrm{MPE}}(\mathcal{E})$ returns $\mathcal{S}_{\mathcal{E}}=\langle\{p\},\{q \wedge r\},\{s\}\rangle$.

The stratification makes it explicit that if a formula (statement) is more primed or more entailed, then it should be more preferred in a merged result than other formulae. Therefore, it has the obvious advantage over using a merging operator that gives a flat base. This is especially useful when knowledge bases are inconsistent.

Example 4. (Continue Example 1) Let $\mathcal{E}=\left\{K_{A}, K_{B}, K_{C}, K_{D}\right\}$, then the stratified base from the algorithm is $\mathcal{S}_{\mathcal{E}}=\langle\{p\},\{q\},\{\neg p, s, q \wedge r\}\rangle$ which clearly shows that $p$ gathers more support from these sources.

Algorithm $\mathrm{S}_{\mathrm{MPE}}(\mathcal{E})$ can be modified to stratify a knowledge profile based on the criteria of the most primed (resp. the most entailed formulae) only by using value Sup ${ }_{1}$ (resp. Sup $_{1}$ ) alone. The following example reveals the subtle difference between the algorithm and its variants.

Example 5. Let $\mathcal{E}=\left\{K_{1}, K_{2}, K_{3}, K_{4}\right\}$ where $K_{1}=\{p, q \wedge r\}, K_{2}=\{p, q \wedge r, s\}$, $K_{3}=\{p, q, s\}$, and $K_{4}=\{p, q, s\}$. The stratification of $\mathcal{E}_{\cup}$ from the exact algorithm is $\mathcal{S}_{\mathcal{E}}=\langle\{p\},\{q\},\{s\},\{q \wedge r\}\rangle$. The stratification of $\mathcal{E}_{\cup}$ when only the most primed

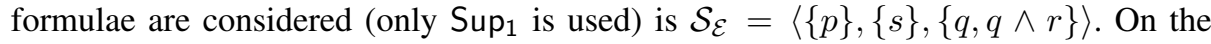
other hand, when only the most entailed formulae are used (only Sup 2 is considered), the result is $\mathcal{S}_{\mathcal{E}}=\langle\{p, q\},\{s\},\{q \wedge r\}\rangle$. 
Definition 11. Let $\mathcal{E}$ be a knowledge profile and every knowledge base in $\mathcal{E}$ be consistent. We define an ordering relation $\preceq \mathrm{MPE}$ on $\mathcal{E}_{\cup}$ induced by $\mathcal{S}_{\mathcal{E}}$ from $\mathrm{S}_{\mathrm{MPE}}(\mathcal{E})$ as $\phi \preceq_{\mathrm{MPE}} \psi$, iff $\phi \in S_{i}, \psi \in S_{j}$, and $i \leq j$.

Proposition 2. Let $\preceq_{\mathrm{MPE}}$ be defined as above, then $\phi \preceq \mathrm{MPE} \varphi$ iff one of the following conditions is true

$$
\begin{aligned}
& S_{\mathcal{E}}^{d p}(\phi)=S_{\mathcal{E}}^{d}(\phi)=S_{\mathcal{E}}^{d p}(\varphi)=S_{\mathcal{E}}^{d}(\varphi) \\
& S_{\mathcal{E}}^{d}(\phi)=S_{\mathcal{E}}^{d}(\varphi)>S_{\mathcal{E}}^{d p}(\varphi) \\
& S_{\mathcal{E}}^{d}(\phi)>S_{\mathcal{E}}^{d}(\varphi) .
\end{aligned}
$$

Conditions 1 and 3 correspond exactly to the if and else statements of lines 8 and line 15 respectively. For Condition 2 , when $S_{\mathcal{E}}^{d p}(\phi)=S_{\mathcal{E}}^{d}(\phi)$, it has the same effect as Condition 3, i.e., $\phi$ is one stratum lower than $\varphi$; however when $S_{\mathcal{E}}^{d p}(\phi)<S_{\mathcal{E}}^{d}(\phi)$, it has the same effect as Condition 1, i.e., $\phi$ and $\varphi$ are in the same stratum. For instance, in Example 5, we have $S_{\mathcal{E}}^{d}(p)=S_{\mathcal{E}}^{d}(q)>S_{\mathcal{E}}^{d p}(q)$ (and $S_{\mathcal{E}}^{d}(p)=S_{\mathcal{E}}^{d p}(p)$ ), so Condition 2 is met and $p$ is ranked ahead of $q$.

Definition 12. Let $\preceq_{\mathrm{MPE}}^{p}$ be a variant of $\preceq_{\mathrm{MPE}}$ on $\mathcal{E}_{\cup}$ such that $\mathrm{S}_{\mathrm{MPE}}(\mathcal{E})$ considers only the most primed formulae in $\mathcal{E}_{\cup}$ for stratification.

Proposition 3. Let $\preceq_{p}$ be as defined in Definition 8, then $\preceq_{\mathrm{MPE}}^{p} \equiv \preceq_{p}$.

Definition 13. Let $\preceq_{\mathrm{MPE}}^{e}$ be a variant of $\preceq_{\mathrm{MPE}}$ on $\mathcal{E}_{\cup}$ such that $\mathrm{S}_{\mathrm{MPE}}(\mathcal{E})$ considers only the most entailed formulae in $\mathcal{E}_{\cup}$ for stratification.

Proposition 4. Let $\preceq_{e}$ be as defined in Definition 10, then $\preceq_{\mathrm{MPE}}^{e} \equiv \preceq_{e}$.

Propositions 3 and 4 show that there is a stratification method corresponding to each of the two total preorder relations defined in Section 3.

\section{Merging Operators based on Stratification}

The result of $\mathrm{S}_{\mathrm{MPE}}(\mathcal{E})$ is a stratified base which can be inconsistent. This base can also be viewed as a prioritized observation base [DDL06] where observations in $S_{i}$ have higher priorities than observations in $S_{j}$ for $j>i$. To obtain a consistent subset from $\mathrm{S}_{\mathrm{MPE}}(\mathcal{E})$, we need to have suitable operators applicable to a stratified base. We define two such operators here and call them merging operators. Let $\mathcal{E}$ be a knowledge profile, and $K=\mathcal{E}_{\cup}$, then $\mathcal{S}_{\mathcal{E}}=\mathcal{S}_{K}=\left\langle S_{1}, \ldots, S_{n}\right\rangle$ denotes its stratification. Let $A=\left\langle A_{1}, \ldots, A_{n}\right\rangle$ such that $A_{i} \subseteq S_{i}$, we define $A_{\cup}=A_{1} \cup \ldots \cup A_{n}$.

\subsection{New merging operators}

Definition 14. Let $\mathcal{E}$ be a knowledge profile and $\mathcal{S}_{\mathcal{E}}=\left\langle S_{1}, \ldots, S_{n}\right\rangle$ be its stratification. Let $A=\left\langle A_{1}, \ldots, A_{n}\right\rangle$ be a subset of $\mathcal{S}_{\mathcal{E}}$ such that $A_{i} \subseteq S_{i}$ and $A_{\cup} \forall \perp$. A lexicographical maximal merging operator, denoted as $\Delta_{\text {leximax }}$, is defined as

$$
\begin{aligned}
\Delta_{\text {leximax }}\left(\mathcal{S}_{\mathcal{E}}\right)=\bigvee\{ & \left\{\cup \mid \text { iffor any } A^{\prime}=\left\langle A_{1}^{\prime}, \ldots, A_{n}^{\prime}\right\rangle, A_{\cup}^{\prime} \forall \perp,\right. \text { then } \\
& \text { either } \forall i\left|A_{i}\right|=\left|A_{i}^{\prime}\right|, \\
& \text { or } \left.\exists i \text {, s.t., }\left|A_{i}\right|>\left|A_{i}^{\prime}\right| \text { and for } j<i,\left|A_{j}\right|=\left|A_{j}^{\prime}\right|\right\}
\end{aligned}
$$


When there is only one stratum in $\mathcal{S}_{\mathcal{E}}$, merging operator $\Delta_{\text {leximax }}\left(\mathcal{S}_{\mathcal{E}}\right)$ is equivalent to operator $\mathrm{Comb}_{4}(\mathcal{E}, \top)$ in [BKMS92] and operator $\Delta_{\top}^{C 4}(\mathcal{E})$ in [Kon00] (when we let the integrity constraint $I C$ be a tautology $\top$ ). However, when $\mathcal{S}_{\mathcal{E}}$ has more than one stratum, $\Delta_{\text {leximax }}\left(\mathcal{S}_{\mathcal{E}}\right)$ preserves more information.

Example 6. Let $\mathcal{E}=\left\{K_{1}, \ldots, K_{5}\right\}$ where $K_{1}=\{\neg p, q\}, K_{2}=\{p, q\}, K_{3}=\{p, q \rightarrow$ $r\}, K_{4}=\{\neg p, s\}$, and $K_{5}=\{\neg p, \neg s\}$. Then $\mathcal{S}_{\mathcal{E}}=\langle\{\neg p\},\{p, q\},\{q \rightarrow r, s, \neg s\}\rangle$. Applying operator $\Delta_{\text {leximax }}$, we have $\Delta_{\text {leximax }}\left(\mathcal{S}_{\mathcal{E}}\right)=\vee\{\{\neg p, q, q \rightarrow r, s\},\{\neg p, q, q \rightarrow$ $r, \neg s\}\}$ which is equivalent to $\{\neg p, q, q \rightarrow r\}$.

Definition 15. Let $\mathcal{E}$ be a knowledge profile and $\mathcal{S}_{\mathcal{E}}=\left\langle S_{1}, \ldots, S_{n}\right\rangle$ be its stratification. Let $A=\left\langle A_{1}, \ldots, A_{n}\right\rangle$ be a subset of $\mathcal{S}_{\mathcal{E}}$ such that $A_{i} \subseteq S_{i}$ and $A_{\cup}$ is consistent. Let $\operatorname{Inc}\left(\mathcal{S}_{\mathcal{E}}\right)=i$. A maximal-consistency based merging operator $\Delta_{\text {conmax }}$, is defined as

$$
\begin{gathered}
\Delta_{\text {conmax }}\left(\mathcal{S}_{\mathcal{E}}\right)=\bigvee\left\{A_{\cup} \mid \cup_{j=1}^{i-1} S_{j} \subseteq A_{\cup}, \text { s.t. }, \forall A^{\prime}=\left\langle A_{1}^{\prime}, \ldots, A_{n}^{\prime}\right\rangle, A_{\cup}^{\prime} \forall \perp,\right. \\
\text { if } \left.\cup_{j=1}^{i-1} S_{j} \subseteq A_{\cup}^{\prime} \text { then }\left|A_{\cup}\right| \geq\left|A_{\cup}^{\prime}\right|\right\}
\end{gathered}
$$

This operator guarantees that all the more preferred consistent formulae are selected first, before considering any further formulae. $\Delta_{\text {conmax }}$ and $\Delta_{\text {leximax }}$ are equivalent when $\mathcal{S}_{\mathcal{E}}$ has only one stratum, i.e., $\mathcal{S}_{\mathcal{E}}=\left\langle\mathcal{E}_{\cup}\right\rangle$. Furthermore, we define $\Delta_{\text {conmax }}^{i}$ as a variant of $\Delta_{\text {conmax }}$ such that $\operatorname{lnc}\left(\mathcal{S}_{\mathcal{E}}\right)=i$ and $\Delta_{\text {conmax }}^{i}=S_{1} \cup \ldots \cup S_{i-1}$.

Example 7. Let a stratified knowledge profile be $\mathcal{S}_{\mathcal{E}}=\langle\{\neg p, q\},\{q \rightarrow r, s, \neg r, \neg r \wedge$ $q\},\{r\}\rangle$. Then $\Delta_{\text {leximax }}\left(\mathcal{S}_{\mathcal{E}}\right)=\{\neg p, q, s, \neg r, \neg r \wedge q\}$ and $\Delta_{\text {conmax }}\left(\mathcal{S}_{\mathcal{E}}\right)=\vee\{\{\neg p, q$, $s, q \rightarrow r, r\},\{\neg p, q, s, \neg r, \neg r \wedge q\}\}$ which is equivalent to $\{\neg p, q, s\}$. In this case, $\Delta_{\text {leximax }} \vdash \Delta_{\text {conmax }}$. However, these two operators are not comparable in general.

\subsection{Properties}

In [DDL06], three merging operators for a prioritized base ${ }^{2}$ are defined. Among them operator best-out, $*_{b o}\left(\mathcal{S}_{\mathcal{E}}\right)$ is defined as

$$
*_{b o}\left(\mathcal{S}_{\mathcal{E}}\right)=\bigwedge\left(\bigwedge S_{j} \mid j<i, \operatorname{lnc}\left(\mathcal{S}_{\mathcal{E}}\right)=i\right)
$$

for $\mathcal{S}_{\mathcal{E}}=\left\langle S_{1}, \ldots, S_{n}\right\rangle$ where $\bigwedge S_{j}=\bigwedge_{\phi \in S_{j}} \phi$

That is, $\bigwedge S_{j}$ is the conjunction of all formulae in $S_{j}$ and $\bigwedge\left(\bigwedge S_{j}\right)$ is the conjunction of all $\bigwedge S_{j}$ for $j=1, \ldots, i-1$.

Let $\operatorname{Cons}\left(\mathcal{S}_{\mathcal{E}}\right)$ be the set of all consistent subsets of $\mathcal{S}_{\mathcal{E}}$, that is, the set of all stratified subsets $A=\left\langle A_{1}, \ldots, A_{n}\right\rangle$, such that $A_{i} \subseteq S_{i}$ and $A_{\cup}$ is consistent. If $\succ$ is a strict order on set $Y$, then $\operatorname{Max}(\succ, Y)$ is defined as $\operatorname{Max}(\succ, Y)=\{y \in Y \mid \forall z \in Y, z \nsucc y\}$

Definition 16. [DDL06] For $S, S^{\prime} \in \operatorname{Cons}\left(\mathcal{S}_{\mathcal{E}}\right)$, define $S^{\prime} \succ_{\text {discrimin }} S$ iff $\exists k$ such that

(a) $\left\langle S_{1}, \ldots, S_{k}\right\rangle \cap S^{\prime} \supset\left\langle S_{1}, \ldots, S_{k}\right\rangle \cap S$, and

(b) $\forall i<k,\left\langle S_{1}, \ldots, S_{i}\right\rangle \cap S^{\prime}=\left\langle S_{1}, \ldots, S_{i}\right\rangle \cap S$.

Then $*_{\text {discrimin }}\left(\mathcal{S}_{\mathcal{E}}\right)=\bigvee\left\{\bigwedge S, S \in \operatorname{Max}\left(\succ_{\text {discrimin }}, \operatorname{Cons}\left(\mathcal{S}_{\mathcal{E}}\right)\right)\right\}$

\footnotetext{
${ }^{2}$ Note: in their original paper a prioritized base is represented as $\sigma=\langle\sigma(1), \ldots, \sigma(n)\rangle$ where $\sigma(i)$ denotes a set of formulae with rank $k_{i}$ and $\sigma(n)$ contains the highest ranked formulae. In this paper, we let $S_{1}$ (not $S_{n}$ ) denote the set of highest ranked formulae and ignore the rank itself since it is not used in the merging.
} 
Definition 17. [DDL06] For $S, S^{\prime} \in \operatorname{Cons}\left(\mathcal{E}_{\cup}\right)$, define $S^{\prime} \succ_{\text {leximin }} S$ iff $\exists k$ such that

(a) $\left|\left\langle S_{1}, \ldots, S_{k}\right\rangle \cap S^{\prime}\right|>\left|\left\langle S_{1}, \ldots, S_{k}\right\rangle \cap S\right|$, and

(b) $\forall i<k$, $\left|\left\langle S_{1}, \ldots, S_{i}\right\rangle \cap S^{\prime}\right|=\left|\left\langle S_{1}, \ldots, S_{i}\right\rangle \cap S\right|$.

Then $*_{\text {leximin }}\left(\mathcal{S}_{\mathcal{E}}\right)=\bigvee\left\{\bigwedge S, S \in \operatorname{Max}\left(\succ_{\text {leximin }}, \operatorname{Cons}\left(\mathcal{S}_{\mathcal{E}}\right)\right)\right\}$

The following logical properties ${ }^{3}$ are given in [DDL06] on merging operators $*$ for prioritized bases.

(PMon) for $i<n, *\left(\left\langle S_{1}, \ldots, S_{i+1}\right\rangle\right) \vdash *\left(\left\langle S_{1}, \ldots, S_{i}\right\rangle\right)$

(Succ) $*\left(\mathcal{S}_{\mathcal{E}}\right) \vdash *\left(\mathcal{S}_{1}\right)$

(Cons) $*\left(\mathcal{S}_{\mathcal{E}}\right)$ is consistent

(Taut) $*\left(\mathcal{S}_{\mathcal{E}}, \top\right) \equiv *\left(\mathcal{S}_{\mathcal{E}}\right)$

(Opt) if $\wedge \mathcal{S}_{\mathcal{E}}$ is consistent then $*\left(\mathcal{S}_{\mathcal{E}}\right) \equiv \wedge \mathcal{S}_{\mathcal{E}}$

(IS) If $\mathcal{S}_{\mathcal{E}_{1}} \approx \mathcal{S}_{\mathcal{E}_{2}}$ then $*\left(\mathcal{S}_{\mathcal{E}_{1}}\right)=*\left(\mathcal{S}_{\mathcal{E}_{2}}\right)$

$(\mathbf{R A})^{4} *\left(\left\langle S_{1}, \ldots, S_{i}\right\rangle\right)=*\left(*\left(\left\langle S_{1}, \ldots, S_{i-1}\right\rangle\right), S_{i}\right)$

Proposition 5. $\Delta_{\text {leximax }}\left(\mathcal{S}_{\mathcal{E}}\right)$ is equivalent to operator $*_{\text {leximin }}\left(\mathcal{S}_{\mathcal{E}}\right)$ and variant $\Delta_{\text {conmax }}^{i}$ is equivalent to $*_{b o}\left(\mathcal{S}_{\mathcal{E}}\right)$.

Proposition 6. Merging operators $\Delta_{\text {leximax }}\left(\mathcal{S}_{\mathcal{E}}\right)$ and $\Delta_{\text {conmax }}^{i}\left(\mathcal{S}_{\mathcal{E}}\right)$ satisfy all the seven properties given above. $\Delta_{\text {conmax }}\left(\mathcal{S}_{\mathcal{E}}\right)$ satisfies (Cons), (Taut), (Opt), (IS) and (RA).

Below we examine how a stratified profile can be used to compare different prioritized merging operators.

Definition 18. Let $\mathcal{S}_{\mathcal{E}}=\left\langle S_{1}, \ldots, S_{n}\right\rangle$ where $\operatorname{Inc}\left(\mathcal{S}_{\mathcal{E}}\right)=i>1$. Let $\Gamma$ be the set of all formula-based merging operators for a prioritized base. Let $\Delta_{1}$ and $\Delta_{2}$ be two operators in $\Gamma$. We define a partial order relation $\preceq$ over $\Gamma$ as: $\Delta_{1} \preceq \Delta_{2}$ iff one of the following conditions holds.

- $S_{1} \cup \ldots \cup S_{i-1} \subseteq \Delta_{1}(\mathcal{E})$ and $S_{1} \cup \ldots \cup S_{i-1} \not \subset \Delta_{2}(\mathcal{E})$;

- $S_{1} \cup \ldots \cup S_{i-1} \subseteq \Delta_{1}(\mathcal{E})$ and $S_{1} \cup \ldots \cup S_{i-1} \subseteq \Delta_{2}(\mathcal{E})$, then $\left|\Delta_{1}(\mathcal{E})\right|>\left|\Delta_{2}(\mathcal{E})\right|$.

$\Delta_{1} \preceq \Delta_{2}$ indicates that $\Delta_{1}$ is at least as efficient as $\Delta_{2}$ to merge a knowledge profile. Here $|\Delta(\mathcal{E})|$ denotes the cardinality of merging result $\Delta(\mathcal{E})$. The first condition says that if an operator can select all the more preferred and consistent formulae while another cannot, then the former is a better merging operator. The second conditions reveals that when all the more preferred and consistent formulae are included, the operator with more additional formulae is better than the other.

\footnotetext{
${ }^{3}$ We only have space in this extended abstract to discuss these properties. In the full paper, we consider further properties including the logical properties in [KP98] when we view the input of such an operator as a knowledge profile and the output as a consistent subset without considering the process of stratification in between. Our operators are also compared with the Adjustment and the Maxi-Adjustment algorithms in [B+04].

${ }^{4}$ Note: Given $\mathcal{S}_{\mathcal{E}}=\left\langle S_{1}, \ldots, S_{n}\right\rangle, S_{1}$ has the most reliable formulae and it is equivalent to $\sigma_{n}$ in the original definition of a prioritized base in [DDL06], therefore, the Right Associativity (RA) property looks like a Left Associativity property.
} 
Proposition 7. Based on Definition 18, we have $\Delta_{\text {leximax }}\left(\mathcal{S}_{\mathcal{E}}\right) \preceq \Delta_{\text {conmax }}^{i}\left(\mathcal{S}_{\mathcal{E}}\right)$ and $*_{\text {leximin }}\left(\mathcal{S}_{\mathcal{E}}\right) \preceq *_{\text {discrimin }}\left(\mathcal{S}_{\mathcal{E}}\right) \preceq *_{b o}\left(\mathcal{S}_{\mathcal{E}}\right)$. $\Delta_{\text {conmax }}\left(\mathcal{S}_{\mathcal{E}}\right)$ is not comparable with $\Delta_{\text {leximax }}\left(\mathcal{S}_{\mathcal{E}}\right)$ or $*_{\operatorname{discrimin}}\left(\mathcal{S}_{\mathcal{E}}\right)$.

Operator $\Delta_{\text {conmax }}\left(\mathcal{S}_{\mathcal{E}}\right)$ does not really take into account the priorities of formulae. Therefore, although it may contain more formulae than other operators, such as, $\Delta_{\text {leximax }}\left(\mathcal{S}_{\mathcal{E}}\right)$, it is less desirable for a prioritized merging.

\section{Related Work and Conclusion}

Approaches to stratifying a knowledge base with default rules have been reported in several research proposals [Pea90,GP91,Bre89,Cho94], all of which are about stratifying a single knowledge base with defaults (rules and/or facts). Since we start with multiple original knowledge bases and aim to merge them into a single knowledge base with priorities automatically generated, these proposals cannot be applied. The priorities of formulae are calculated based on the degree of support they receive from the input knowledge bases. A knowledge profile is then stratified based on the priorities of formulae in the profile, with formulae having the highest priority in the current profile being the most preferred formulae. In this respect, our idea of stratification is in spirit similar to Pearl's method in [Pea90], that is, the more support a formula (rule) gets, the higher rank it is assigned.

Our method on stratification has some similarities with voting systems. In a voting system, many voting policies require that a voter simply votes for the chosen candidate(s) without requiring preferences over the chosen candidates. In plurality voting, a voter is allowed to vote for one candidate only, so such a knowledge base contains one formula (i.e., candidate). In approval voting, a voter can vote for multiple candidates without preferring one over the other, so such a knowledge base contains multiple formulae. For both cases, when our algorithm is applied to stratify a set of votes (knowledge bases), the algorithm produces the same result as either of the two voting policies. More specifically, in both voting policies, the candidates who receive the largest number of votes are the winners (at least for the current round, if a single winner has to be selected, more rounds of votes are required). These candidates are exactly the formulae selected in the first stratum in our algorithm. When this stratum contains a single formula, a single winner is selected. Therefore, let $\mathcal{E}$ be a knowledge profile and each knowledge base in $\mathcal{E}$ contains votes from a voter following the voting rules in plurality (resp. approval) voting. Then the first stratum from $\mathrm{S}_{\mathrm{MPE}}(\mathcal{E})$ is equivalent to the result of plurality (resp. approval) voting system.

In conclusion, in this paper, we focused on how to extract information provided by the original sources about which formulae gathered more support. This information is preserved in the form of a stratified base for formulae in the union of original bases. Stratifying a knowledge base in this way overcomes the problem of deciding which formula should be kept when a choice has to be made to resolve a conflict after merging. An obvious decision is that a higher ranked formula shall be kept. Also, such a merged base provides a basis for ranking merging operators such as a merging operator that preserve as many high ranked formulae as possible is certainly better than the one that cannot. 


\section{References}

[BKMS92] C Baral, S Kraus, J Minker, V Subrahmanian 1992. Combining knowledge bases consisting of first-order theories. Computational Intelligence 8(1): 45-71.

[BB04] S Benferhat and R Baida 2004. A stratified first order logic approach for access control. Int. J. of Int. Sys. 19:817-836.

[B+93] S Benferhat, C Cayrol, D Dubois, J Lang and H Prade 1993. Inconsistency management and prioritized syntax-based entailment. Proc. of IJCAI'93:640-645.

[BDP96] S Benferhat, D Dubois and H Prade 1996. Reasoning in inconsistent stratified knowledge bases. Proc. of ICMVL'96:184-189.

[B+98] S Benferhat, D Dubois, J Lang, H Prade, A Saffiotti and Ph Smets 1998. A General approach for inconsistency handling and merging information in prioritized knowledge bases. Proc. of KR'98:466-477.

[B+04] S Benferhat, S Kaci, D Berre and M Williams 2004. Weakening conflicting information for iterated revision and knowledge integration. Art. Int. 153(1-2):339-371.

[BLR07] S Benferhat, S Lagrue, and J Rossit. 2007. An Egalitarist Fusion of Incommensurable Ranked Belief Bases under Constraints. Proc. of AAAI'07, 367-372.

[Bre89] G Brewka 1989. Preferred subtheories - an extended logical framework for default reasoning. Proc. of IJCAI'89:1043-1048.

[Bre04] G Brewka 2004. A rank-based description language for qualitative preferences. Proc. of ECAI'04:303-307.

[CD97] M Cadoli and F Donini 1997. A survey on knowledge compilation. AI Communication 10(3-4): 137-150.

[Cho94] P Cholewinski 1994. Stratified default logic. Proc. of Computer Science Logic'94:456-470, Springer LNCS 933.

[Dav05] A Davis 2005. Just Enough Requirements Management: Where Software Development Meets Marketing. New York: Dorset House.

[DDL06] J Delgrande, D Dubois, and J Lang 2006. Iterated revision as prioritized merging. Proc. of KR06:201-220.

[EKM05] P Everaere, S Konieczny and P Marquis 2005. Quota and Gmin merging operators. Proc. of IJCAI'05:424-429.

[Gad88] P Gädenfors 1988. Knowledge in Flux-Modeling the Dynamic of Epistemic States. MIT Press, Cambridge.

[GP91] M Goldszmidt and J Pearl 1991. System $Z^{+}$: A formalism for reasoning with variable-strength defaults. Proc. of AAAI'91:399-404.

[KG06] Y Katz and J Golbeck 2006. Social Network-based Trust in Prioritized Default Logic. Proc. of AAAI'06.

[KLM04] S Konieczny, J Lang and P Marquis 2004. D $A^{2}$ operators. Art. Int. Vol. 157(1-2): 49-79.

[Kon00] S Konieczny 2000. On the difference between merging knowledge bases and combining them. KR'O0 12-17.

[KP98] S Konieczny and R Pino Pérez 1998. On the logic of merging. In Proc. of KR98:488498.

[Pea90] J Pearl 1990. System Z: A natural ordering of defaults with tractable applications to default reasoning. Proc. of the 3rd Int. Conf. on Theor. Aspects of Reas. about Know::121-135. 